\title{
STABILITY AND BIFURCATION ANALYSIS OF A PIPE CONVEYING PULSATING FLUID WITH COMBINATION PARAMETRIC AND INTERNAL RESONANCES
}

\author{
Liangqiang Zhou ${ }^{1}$, Fangqi Chen ${ }^{1}$, Yushu Chen ${ }^{2}$ \\ ${ }^{1}$ Department of Mathematics \\ Nanjing University of Aeronautics and Astronautics, Nanjing 210016, \\ PR China \\ ${ }^{2}$ Department of Mechanics \\ Tianjin University, Tianjin, 300072, PR China \\ zlqrex@sina.com, fangqichen@nuaa.edu.cn
}

\begin{abstract}
The stability and bifurcations of a hinged-hinged pipe conveying pulsating fluid with combination parametric and internal resonances are studied with both analytical and numerical methods. The system has geometric cubic nonlinearity. Three types of critical points for the bifurcation response equations are considered. These points are characterized by a double zero and two negative eigenvalues, double zero and a pair of purely imaginary eigenvalues, and two pairs of purely imaginary eigenvalues, respectively. With the aid of normal form theory, the expressions for the critical bifurcation lines leading to incipient and secondary bifurcations are obtained. Possible bifurcations leading to 2-D tori are also investigated. Numerical simulations confirm the analytical results.
\end{abstract}

Keywords- Pipe with Pulsating Fluid, Nonlinear Vibration, Perturbation Methods, Parametric Resonances, Stability, Bifurcation;

\section{INTRODUCTION}

The linear and nonlinear dynamics of pipes conveying fluid has been studied widely during the last decades. Detailed review and extensive bibliography on this flowinduced vibrations and instabilities of piping and cylindrical structures were provided by Padoussis et al [1-3]. The parametric instabilities depending on the amplitude and frequency of flow fluctuation will occur when the flow velocity has a harmonically fluctuating component over a mean value. A lot of investigations based on linearized analytical models of these parametric instability problems for simply supported pipes were done by Chen [4], Padoussis and Issid[5], Padoussis and Sundararajan [6], Ginsberg [7] and Ariaratnam and Namachchivaya [8], Jayaraman and Tien [9]. They studied the parametric and combination resonances and evaluated instability with numerical methods. In [10], Panda and Kar studied the nonlinear dynamics of a hingedhinged pipe conveying pulsating fluid subjected to combination and principle parametric resonance in the presence of internal resonance with the method of multiple scales and numerical methods. Using the method of multiple scales, Panda and Kar [11] studied the nonlinear planar vibration of a pipe conveying pulsating fluid subjected to principal parametric resonance in the presence of internal resonance. By considering the effect of motion constraints modeled as cubic springs, the nonlinear dynamics of simply 
supported pipes conveying pulsating fluid was further investigated, and some new dynamical behaviors including quasi-periodic and chaotic motions were obtained [12]. Jin and Song [13] investigated the stability and parametric resonances of supported pipes conveying pulsating with numerical methods. The post-divergence behavior of extensible fluid-conveying pipes supported at both ends was studied by ModarresSadeghi and Padoussis [14], and a supercritical pitchfork bifurcation was obtained. Using numerical methods, Wang and $\mathrm{Ni}$ [15] investigated the stability and chaotic motions of a standing pipe conveying fluid. A spectral element model was developed for the uniform straight pipelines conveying internal unsteady fluid [16]. By using the Melnikov method, the global dynamics of parametrically excited conveying fluid near 0:1 resonance was studied, and chaotic dynamics may exist in the system [17]. The stability and dynamics of a cantilevered pipe conveying fluid with motion-limiting constraints and a linear spring support were investigated [18]. Using the Euler-Bernoulli beam theory and nonlinear Lagrange strain theory, a new nonlinear model of a straight pipe conveying fluid was presented [19]. The vibration was analyzed with the Galerkin method. Using Hamilton's principle and Galerkin method, Sina [20] investigated the non-linear vibrations of slightly curved pipes conveying fluid with constant velocity. Periodic and chaotic motions were observed in the transverse vibrations.

In this paper, the stability and bifurcations of a hinged-hinged pipe conveying pulsating fluid with combination parametric and internal resonances are studied both analytically and numerically. Three types of critical points for the bifurcation response equations are discussed. These points are characterized by a double zero and two negative eigenvalues, double zero and a pair of purely imaginary eigenvalues, and two pairs of purely imaginary eigenvalues, respectively. With the aid of normal form theory, the expressions for the critical bifurcation lines leading to incipient and secondary bifurcations are obtained. Possible bifurcation solutions and their stability are investigated. Numerical simulations are also given, which verify the analytical results.

\section{FORMULATION OF THE PROBLEM}

Consider a uniform horizontal pipe hinged at both ends conveying fluid with a flow-velocity having harmonically pulsating component superimposed over a steady one (Fig 1). Assume that the motion is planar and the uniform cross-section remains plane during the motion and the tube behaves like an Euler-Bernoulli beam in transverse vibration. It is also assumed that the fluid is incompressible and has plug flow conditions. The equation of transverse motion of the pipe including the nonlinearity due to midline stretching is

$$
\begin{aligned}
& E I \frac{\partial^{4} y}{\partial x^{4}}+E^{*} I \frac{\partial^{5} y}{\partial x^{4} \partial t}+2 M U \frac{\partial^{2} y}{\partial x \partial t}+(M+m) \frac{\partial^{2} y}{\partial t^{2}}+c \frac{\partial y}{\partial t} \\
& +\left[M U^{2}-\bar{T}+M \frac{\partial U}{\partial t}(L-x)-\frac{E A}{2 L} \int_{0}^{L}\left(y^{\prime}\right)^{2} \mathrm{~d} x-\frac{E^{*} A}{L} \int_{0}^{L}\left(y^{\prime} \dot{y}^{\prime}\right) \mathrm{d} x\right] \frac{\partial^{2} y}{\partial x^{2}}=0
\end{aligned}
$$

with the boundary conditions

$$
y(0, t)=y(L, t)=\frac{\partial^{2} y}{\partial x^{2}}(0, t)=\frac{\partial^{2} y}{\partial x^{2}}(L, t)=0
$$

where $x$ is the longitudinal coordinate, $y$ is the transverse deflection, $\bar{T}$ is the 
externally imposed axial tension, $m$ and $M$ are the mass per unit length of pipe and fluid materials, respectively, $A$ is the cross sectional area of the pipe, $L$ is the length, $E I$ is the flexural stiffness of the pipe material, $E^{*}$ is the coefficient of internal dissipation of the pipe material which is assumed to be viscoelastic and of the KelvinVoigt type and $c$ is the external damping factor, $U$ is the fluid velocity which has the following form

$$
U=U_{0}(1+v \sin \omega t)
$$

where $U_{0}$ is the mean flow velocity, $v$ and $\omega$ are the amplitude and frequency of the flow-velocity fluctuation, which may lead to parametric instabilities.

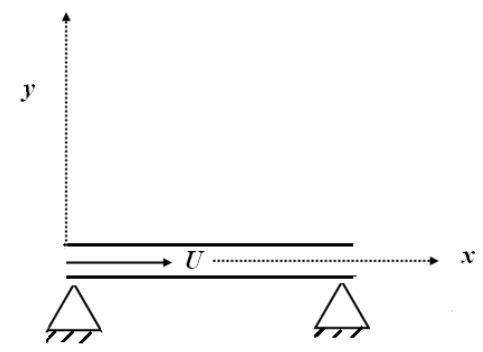

Figure 1. Schematics of the model.

Introducing the following dimensionless quantities

$$
\begin{aligned}
& \xi=\frac{x}{L}, \quad w=\frac{y}{L}, \quad \tau=\frac{1}{L^{2}}\left(\frac{E I}{M+m}\right)^{1 / 2} t=\omega_{n} t, \quad u=\left(\frac{M}{E I}\right)^{1 / 2} U L, \quad \beta=\frac{M}{M+m}, \quad \Gamma=\frac{\bar{T} L^{2}}{E I} \\
& , \quad \sigma^{*}=\frac{c L^{2}}{\sqrt{E I(M+m)}}, \quad \alpha^{*}=\frac{E^{*}}{L^{2}}\left\{\frac{I}{(M+m) E}\right\}^{1 / 2}, \quad k=\frac{A L^{2}}{2 I}, \quad \Omega=\frac{\omega}{\omega_{n}} \\
& \delta^{*}=\frac{E^{*} A}{\sqrt{E I(M+m)}},
\end{aligned}
$$

the equation of motion becomes [10]

$$
\begin{aligned}
& \alpha^{*} w^{\prime \prime \prime}+w^{\prime \prime \prime}+\left\{u^{2}-\Gamma+\beta^{1 / 2} \dot{u}(1-\xi)\right\} w^{\prime \prime}+2 \beta^{1 / 2} u \dot{w}^{\prime}+\sigma^{*} \dot{w}+\ddot{w} \\
& \quad-k \int_{0}^{1}\left(w^{\prime}\right)^{2} \mathrm{~d} \xi w^{\prime \prime}-\delta^{*} \int_{0}^{1} w^{\prime} \dot{w}^{\prime} \mathrm{d} \xi w^{\prime \prime}=0, \\
& u=u_{0}(1+v \sin \Omega \tau),
\end{aligned}
$$

The primes and dots represent differentiation with respect to non-dimensional longitudinal coordinate $\xi$ and non-dimensional time $\tau$. To express the smallness of the amplitude of motion $w$, we scale it with the factor $\varepsilon^{1 / 2}$ as in [10], where the small parameter $\varepsilon$ is a measure of amplitude and is also used as a book keeping device in the subsequent perturbation analysis. Introducing this scaling factor and using Eq.(6) for pulsating flow velocity, the non-dimensional equation of motion can be written as

$$
\begin{aligned}
& w^{\prime \prime \prime}+\left(u_{0}^{2}+2 \varepsilon u_{0} u_{1} \sin \Omega \tau-\Gamma\right) w^{\prime \prime}+\sqrt{\beta} \varepsilon u_{1} \Omega \cos \Omega \tau(1-\xi) w^{\prime \prime}+2 \sqrt{\beta}\left(u_{0}+\varepsilon u_{1} \sin \Omega \tau\right) \dot{w}^{\prime} \\
& \quad+2 \varepsilon \mu \dot{w}+2 \varepsilon \alpha \dot{w}^{\prime \prime \prime}+\ddot{w}=\varepsilon k \int_{0}^{1}\left(w^{\prime}\right)^{2} \mathrm{~d} \xi w^{\prime \prime}+O\left(\varepsilon^{2}\right),
\end{aligned}
$$

with the associated boundary conditions

$$
w(0, \tau)=w(1, \tau)=w^{\prime \prime}(0, \tau)=w^{\prime \prime}(1, \tau)=0,
$$


where

$$
\mu=\sigma^{*} / 2 \varepsilon, \quad \alpha=\alpha^{*} / 2 \varepsilon, \quad u_{0} v=\varepsilon u_{1}
$$

Using the method of multiple scales, introducing the time scale $T_{n}=\varepsilon^{n} \tau, n=0,1, \cdots$, and the time derivatives $\frac{\mathrm{d}}{\mathrm{d} \tau}=D_{0}+\varepsilon D_{1}+\cdots, \frac{\mathrm{d}^{2}}{\mathrm{~d} \tau^{2}}=D_{0}^{2}+2 \varepsilon D_{0} D_{1}+\cdots, D_{n}=\frac{\partial}{\partial T_{n}}$, $n=0,1, \cdots$, we write the expansion of $w(\xi, \tau)$ in the form

$$
w(\xi, \tau)=w_{0}\left(T_{0}, T_{1}, \xi\right)+\varepsilon w_{1}\left(T_{0}, T_{1}, \xi\right)+\cdots
$$

Substituting Eq.(10) into (7) and (8), and equating coefficients of like powers of $\varepsilon$ on both sides, one can obtain

$$
\begin{gathered}
O\left(\varepsilon^{0}\right): D_{0}^{2} w_{0}+2 \sqrt{\beta} u_{0} D_{0} w_{0}^{\prime}+\left(u_{0}^{2}-\Gamma\right) w_{0}^{\prime \prime}+w_{0}^{\prime \prime \prime \prime}=0, \\
w_{0}(0, \tau)=w_{0}(1, \tau)=w_{0}^{\prime \prime}(0, \tau)=w_{0}^{\prime \prime}(1, \tau)=0, \\
O\left(\varepsilon^{1}\right): D_{0}^{2} w_{1}+2 \sqrt{\beta} u_{0} D_{0} w_{1}^{\prime}+\left(u_{0}^{2}-\Gamma\right) w_{1}^{\prime \prime}+w_{1}^{\prime \prime \prime} \\
=-2 D_{0} D_{1} w_{0}-2 \alpha D_{0} w_{0}^{\prime \prime \prime}-2 \mu D_{0} w_{0}-2 \sqrt{\beta} u_{0} D_{1} w_{0}^{\prime}-2 \sqrt{\beta} u_{1} \sin \Omega T_{0} \times D_{0} w_{0}^{\prime} \\
-\sqrt{\beta} u_{1} \Omega \cos \Omega T_{0}(1-\xi) w_{0}^{\prime \prime}-2 \sqrt{\beta} u_{0} u_{1} \sin \Omega T_{0} \times D_{0} w_{0}^{\prime}+k w_{0}^{\prime \prime} \int_{0}^{1} w_{0}^{\prime 2} \mathrm{~d} x, \\
w_{1}(0, \tau)=w_{1}(1, \tau)=w_{1}^{\prime \prime}(0, \tau)=w_{1}^{\prime \prime}(1, \tau)=0
\end{gathered}
$$

According to (11), we can write

$$
w_{0}\left(T_{0}, T_{1}, \xi\right)=A_{1}\left(T_{1}\right) \phi_{1}(\xi) e^{\mathrm{i} \omega_{1} T_{0}}+A_{2}\left(T_{1}\right) \phi_{2}(\xi) e^{\mathrm{i} \omega_{2} T_{0}}+c c,
$$

where the complicate expressions of $\phi_{m}(\xi)(m=1,2)$ are given in reference [10]. Substituting (13) into (12), considering the case of the internal resonance and combination parametric resonance, i.e.,

$$
\omega_{2}=3 \omega_{1}+\varepsilon \sigma_{1}, \quad \Omega=\omega_{1}+\omega_{2}+\varepsilon \sigma_{2},
$$

the modulation equations can be written as

$$
\begin{aligned}
& 2 A_{1}^{\prime}+2 \mu C_{1} A_{1}+2 \alpha e_{1} A_{1}+8 S_{1} A_{1}^{2} \bar{A}_{1}+8 S_{2} A_{1} A_{2} \bar{A}_{2}+8 G_{1} \bar{A}_{1}^{2} A_{2} e^{\mathrm{i} \sigma_{1} T_{1}}+2 H_{4} \bar{A}_{2} e^{\mathrm{i} \sigma_{2} T_{2}}=0, \\
& 2 A_{2}^{\prime}+2 \mu C_{2} A_{2}+2 \alpha e_{2} A_{2}+8 S_{4} A_{2}^{2} \bar{A}_{2}+8 S_{3} A_{1} A_{2} \bar{A}_{1}+8 G_{2} \bar{A}_{1}^{3} e^{-\mathrm{i} \sigma_{1} T_{1}}+2 H_{5} \bar{A}_{1} e^{\mathrm{i} \sigma_{2} T_{2}}=0,
\end{aligned}
$$

The coefficients which are very complicated and can be seen in the appendix of reference [10] are omitted here.

$$
\text { Letting } \quad A_{n}=\frac{1}{2}\left[p_{n}\left(T_{1}\right)-\mathrm{i} q_{n}\left(T_{1}\right)\right] e^{i \lambda_{n}\left(T_{1}\right)}, \quad(n=1,2),
$$

substituting it into Eqs.(15) and (16), carrying out algebraic manipulations and separating real and imaginary parts, we can obtain the normalized reduced equations as follows [10]

$$
\begin{aligned}
p_{1}^{\prime}= & -\mu C_{1 R} p_{1}-\mu C_{1 I} q_{1}-\alpha e_{1 R} p_{1}-\alpha e_{1 I} q_{1}-S_{1 R}\left(p_{1}^{3}+p_{1} q_{1}^{2}\right)-S_{1 I}\left(p_{1}^{2} q_{1}+q_{1}^{3}\right) \\
& -S_{2 R}\left(p_{1} p_{2}^{2}+p_{1} q_{2}^{2}\right)-S_{2 I}\left(q_{1} p_{2}^{2}+q_{1} q_{2}^{2}\right)-\vartheta_{1} q_{1}-H_{4 R} p_{2}+H_{4 I} q_{2} \\
& -G_{1 R}\left(p_{1}^{2} p_{2}-p_{2} q_{1}^{2}+2 p_{1} q_{1} q_{2}\right)+G_{1 I}\left(2 p_{1} q_{1} p_{2}-p_{1}^{2} q_{2}+q_{1}^{2} q_{2}\right), \\
q_{1}^{\prime}= & -\mu C_{1 R} q_{1}+\mu C_{1 I} p_{1}-\alpha e_{1 R} q_{1}+\alpha e_{1 I} p_{1}+S_{1 I}\left(p_{1}^{3}+p_{1} q_{1}^{2}\right)-S_{1 R}\left(p_{1}^{2} q_{1}+q_{1}^{3}\right) \\
& -S_{2 R}\left(q_{1} p_{2}^{2}+q_{1} q_{2}^{2}\right)+S_{2 I}\left(p_{1} p_{2}^{2}+p_{1} q_{2}^{2}\right)+\vartheta_{1} p_{1}+H_{4 R} q_{2}+H_{4 I} p_{2} \\
+ & G_{1 R}\left(-p_{1}^{2} q_{2}+q_{1}^{2} q_{2}+2 p_{1} q_{1} p_{2}\right)+G_{1 I}\left(2 p_{1} q_{1} q_{2}+p_{1}^{2} p_{2}-p_{2} q_{1}^{2}\right),
\end{aligned}
$$




$$
\begin{aligned}
p_{2}^{\prime}= & -\mu C_{2 R} p_{2}-\mu C_{2 I} q_{2}-\alpha e_{2 R} p_{2}-\alpha e_{2 I} q_{2}-S_{4 R}\left(p_{2}^{3}+p_{2} q_{2}^{2}\right)-S_{4 I}\left(q_{2}^{3}+p_{2}^{2} q_{2}\right) \\
& -S_{3 R}\left(p_{1}^{2} p_{2}+p_{2} q_{1}^{2}\right)-S_{3 I}\left(p_{1}^{2} q_{2}+q_{1}^{2} q_{2}\right)-\vartheta_{2} q_{2}-H_{5 R} p_{1}+H_{5 I} q_{1} \\
& -G_{2 R}\left(p_{1}^{3}-3 p_{1} q_{1}^{2}\right)+G_{2 I}\left(q_{1}^{3}-3 p_{1}^{2} q_{1}\right), \\
q_{2}^{\prime}= & -\mu C_{2 R} q_{2}+\mu C_{2 I} p_{2}-\alpha e_{2 R} q_{2}-\alpha e_{2 I} p_{2}-S_{4 R}\left(q_{2}^{3}+p_{2}^{2} q_{2}\right)+S_{4 I}\left(p_{2}^{3}+p_{2} q_{2}^{2}\right) \\
& -S_{3 R}\left(p_{1}^{2} q_{2}+q_{1}^{2} q_{2}\right)+S_{3 I}\left(p_{1}^{2} p_{2}+q_{1}^{2} q_{2}\right)+\vartheta_{2} p_{2}+H_{6 R} q_{2}+H_{6 I} p_{2} \\
& +G_{2 R}\left(q_{1}^{3}-3 p_{1}^{2} q_{1}\right)+G_{2 I}\left(p_{1}^{3}-3 p_{1} q_{1}^{2}\right),
\end{aligned}
$$

where $\vartheta_{1}=\left(\sigma_{1}+\sigma_{2}\right) / 4, \quad \vartheta_{2}=\left(3 \sigma_{2}-\sigma_{1}\right) / 4$. point $\left(p_{1}, q_{1}, p_{2}, q_{2}\right)=(0,0,0,0)$ for Eq. (18) is

$$
\lambda^{4}+R_{1} \lambda^{3}+R_{2} \lambda^{3}+R_{3} \lambda+R_{4}=0
$$

where $R_{1}, R_{2}, R_{3}, R_{4}$ are complicated functions of the parameters and omitted here.

According to the Routh-Hurwitz criterion, the initial equilibrium point $\left(p_{1}, q_{1}, p_{2}\right.$, $\left.q_{2}\right)=(0,0,0,0)$ is stable if the following conditions are satisfied.

$$
R_{1}>0, \quad R_{1} R_{2}-R_{3}>0, \quad R_{3}\left(R_{1} R_{2}-R_{3}\right)-R_{1}^{2} R_{4}>0, \quad R_{4}>0 .
$$

\section{BIFURCATION ANALYSIS}

Conditions (21) imply that all the eigenvalues of the Jacobi matrix have negative real parts. When (21) are not satisfied, this is not the case. Three cases will be discussed here.

\subsection{Case 1: Double zero and two negative eigenvalues}

Taking $R_{1}=4, R_{2}=3, R_{3}=R_{4}=0$, Eq.(20) has a double zero and two negative eigenvalues $\lambda_{1,2}=-1, \lambda_{4}=-3$. One choice of parameters that satisfy these conditions is

$$
\begin{aligned}
& \mu=1, \quad C_{1 R}=0, \quad C_{2 R}=2, \quad C_{1 I}=\frac{\sqrt{6 \sqrt{13}+15}}{3}, \quad C_{2 I}=\frac{\sqrt{6 \sqrt{13}+6}}{3}, H_{4 I}=1, \\
& H_{5 I}=\frac{4 \sqrt{13}+10}{3}, \quad \alpha=0, \quad \sigma_{1}=\sigma_{2}=0, \quad H_{4 R}=H_{3 R}=H_{6 R}=H_{6 I}=0 .
\end{aligned}
$$

Let us consider $\sigma_{1}, \sigma_{2}$ as perturbation parameters. Using the parameter transformations $\sigma_{1}=\tilde{\sigma}_{1}, \quad \sigma_{2}=\tilde{\sigma}_{2}$ and the state variable transformation

$$
\left[\begin{array}{l}
p_{1} \\
q_{1} \\
p_{2} \\
q_{2}
\end{array}\right]=\left[\begin{array}{llll}
J_{11} & J_{12} & J_{13} & J_{14} \\
J_{21} & J_{22} & J_{23} & J_{24} \\
J_{31} & J_{32} & J_{33} & J_{34} \\
J_{41} & J_{42} & J_{43} & J_{44}
\end{array}\right]\left[\begin{array}{c}
x_{1} \\
x_{2} \\
x_{3} \\
x_{4}
\end{array}\right]
$$

where $J_{i j}(i, j=1,2,3,4)$ are given in the appendix (A.1), one may transformation Eq.(18) into a new system as follows

$$
\frac{\mathrm{d} x_{1}}{\mathrm{~d} t}=a_{11} x_{1}+a_{12} x_{2}+a_{13} x_{3}+a_{14} x_{4}+N f_{1}
$$




$$
\begin{aligned}
& \frac{\mathrm{d} x_{2}}{\mathrm{~d} t}=a_{21} x_{1}+a_{22} x_{2}+a_{23} x_{3}+a_{24} x_{4}+N f_{2} \\
& \frac{\mathrm{d} x_{3}}{\mathrm{~d} t}=a_{31} x_{1}+a_{32} x_{2}+a_{33} x_{3}+a_{34} x_{4}+N f_{3} \\
& \frac{\mathrm{d} x_{4}}{\mathrm{~d} t}=a_{41} x_{1}+a_{42} x_{2}+a_{43} x_{3}+a_{44} x_{4}+N f_{4}
\end{aligned}
$$

where $a_{i j}(i, j=1,2,3,4)$ are given in the appendix (A.2), $N f_{i}(i=1,2,3,4)$ are third order nonlinear terms whose expressions are very complicated and omitted here.

The Jacobi matrix of Eq.(23) evaluated on the initial equilibrium solution $\left(x_{1}, x_{2}, x_{3}\right.$, $\left.x_{4}\right)=(0,0,0,0)$ at the critical point $P_{c}\left(\tilde{\sigma}_{1 c}=\tilde{\sigma}_{2 c}=0\right)$ is now in the canonical form

$$
J_{\left(x_{i}=0\right)}=\left[\begin{array}{cccc}
0 & 1 & 0 & 0 \\
0 & 0 & 0 & 0 \\
0 & 0 & -1 & 0 \\
0 & 0 & 0 & -3
\end{array}\right]
$$

The local dynamical behavior of system (23) is characterized by the critical variables $x_{1}$ and $x_{2}$. Further more, the bifurcation solutions for the non-critical variables $x_{3}$ and $x_{4}$ may be determined from Eq.(23) up to leading order terms [21]. Therefore one may verify that neglecting $x_{3}$ and $x_{4}$ (i.e., setting $x_{3}=x_{4}=0$ ) in the first two equations of Eq.(23) does not effect the results of the bifurcation solution $\left(x_{1}, x_{2}\right)$ and their stability conditions up to leading order terms. So, in order to consider the bifurcation and stability properties of system (23) in the vicinity of the critical point $P_{c}$, one only needs to analyze the following two-dimensional system:

$$
\begin{aligned}
& \frac{\mathrm{d} x_{1}}{\mathrm{~d} t}=a_{11} x_{1}+a_{12} x_{2}+N f f_{1}, \\
& \frac{\mathrm{d} x_{2}}{\mathrm{~d} t}=a_{21} x_{1}+a_{22} x_{2}+N f f_{2},
\end{aligned}
$$

where $N f f_{i}$ are complicated third order nonlinear terms and omitted. Now based on the reduced system (25), the results and formulae obtained in [22] can be applied here. Using these methods, we can study the stability and bifurcations of this model analytically. Applying the general formula yields the following results.

The stability conditions for the initial equilibrium solution $x_{i}=0$ are described by

$$
131.03 \tilde{\sigma}_{1}-16.86 \tilde{\sigma}_{2}<0 \text { and } 10.61 \tilde{\sigma}_{1}+5.03 \tilde{\sigma}_{2}>0 \text {, }
$$

which leads to two critical lines. One of these is

$$
L_{1}: 10.61 \tilde{\sigma}_{1}+5.03 \tilde{\sigma}_{2}=0,\left(131.03 \tilde{\sigma}_{1}-16.86 \tilde{\sigma}_{2}<0\right),
$$

along which a static bifurcation solution takes place from the initial equilibrium solution and the solution is expressed by

$$
\begin{aligned}
& x_{1}^{2}=-\left(0.028 \tilde{\sigma}_{1}+0.0015 \tilde{\sigma}_{2}\right), \\
& x_{2}=\left(-0.70 \tilde{\sigma}_{1}+1.11 \tilde{\sigma}_{2}\right) x_{1},
\end{aligned}
$$


It is called a pitchfork bifurcation.

On the other hand, the second critical line

$$
L_{2}: 131.03 \tilde{\sigma}_{1}-16.86 \tilde{\sigma}_{2}=0, \quad\left(10.61 \tilde{\sigma}_{1}+5.03 \tilde{\sigma}_{2}>0\right)
$$

describes a dynamic boundary where the initial equilibrium solution loses its stability and bifurcates a family of limit cycles. Again from Table 1 of reference [22], one may find the stability condition for the family of limit cycles, given by

$$
\gamma_{11}-\gamma_{22}=-11.2<0
$$

so the family of limit cycles bifurcating from the initial equilibrium solution is stable.

The static bifurcation solution (28) becomes unstable on the third critical line

$$
L_{3}: 1.11 \tilde{\sigma}_{1}+111.20 \tilde{\sigma}_{2}=0
$$

from which another family of limit cycles which is usually called secondary Hopf bifurcations occurs. The frequency of this family of limit cycles is

$$
\omega_{c}=\sqrt{-\left(0.77 \tilde{\sigma}_{1}+0.12 \tilde{\sigma}_{2}\right)}>0
$$

where $0.77 \tilde{\sigma}_{1}+0.12 \tilde{\sigma}_{2}<0$ since the secondary Hopf bifurcation solution exists in the region located on the right of the critical line $L_{1}$ (see Fig.2). The stability condition for this family of limit cycles is given as follows:

$$
\gamma_{12}+\gamma_{21}=-\frac{0.57}{\omega_{c}}<0
$$

Therefore the secondary Hopf bifurcation solution is stable. The critical bifurcation lines are illustrated in the parameter space in Fig 2. From Fig 2 it is seen that there may exist stable trivial and non-trivial equilibrium solutions, periodic motions in this case.



Figure 2. The bifurcation diagram in the case of double zero and two negative eigenvalues.

\subsection{Case 2:Double zero and a pair of purely imaginary eigenvalues}

Taking the parameters

$$
\begin{aligned}
& \mu=\frac{1}{2}, \quad C_{1 R}=1, \quad C_{2 R}=0, C_{1 I}=\sqrt{\frac{5}{3}}, C_{2 I}=\sqrt{\frac{20}{3}}, H_{4 I}=1, H_{5 I}=\frac{4}{3}, \\
& \alpha=0, \quad \sigma_{1}=\sigma_{2}=0, \quad H_{4 R}=H_{3 R}=H_{6 R}=H_{6 I}=0,
\end{aligned}
$$

in Eq.(20) yields $R_{1}=R_{3}=R_{4}=0, R_{2}=1$, so the eigenvalues of the Jacobian are 
$\lambda_{1,2}=0$, and $\lambda_{3,4}= \pm \mathrm{i}$, where $\mathrm{i}=\sqrt{-1}$. Choosing $\sigma_{1}$ and $\sigma_{2}$ as parameters, and using the parameter transformation $\sigma_{1}=\tilde{\sigma}_{1}, \sigma_{2}=\tilde{\sigma}_{2}$, introducing the following state variable transformation

$$
\left[\begin{array}{l}
p_{1} \\
q_{1} \\
p_{2} \\
q_{2}
\end{array}\right]=\left[\begin{array}{cccc}
0 & \sqrt{15} & 0 & \frac{\sqrt{15}}{5} \\
2 & 3 & 1 & 0 \\
1 & 1 & \frac{1}{2} & \frac{1}{2} \\
\frac{\sqrt{15}}{3} & \sqrt{15} & -\frac{\sqrt{15}}{30} & \frac{\sqrt{15}}{10}
\end{array}\right]\left[\begin{array}{l}
x_{1} \\
x_{2} \\
x_{3} \\
x_{4}
\end{array}\right]
$$

one may obtain the following equations

$$
\begin{aligned}
\frac{\mathrm{d} x_{1}}{\mathrm{~d} t}= & \frac{\sqrt{15}}{12}\left(\tilde{\sigma}_{1}+\tilde{\sigma}_{2}\right) x_{1}+\left(1-\frac{\sqrt{15}}{6} \tilde{\sigma}_{2}\right) x_{2}+\sqrt{15}\left(\frac{1}{15} \tilde{\sigma}_{1}+\frac{7}{60} \tilde{\sigma}_{2}\right) x_{3}+\frac{\sqrt{15}}{60} \tilde{\sigma}_{2} x_{4}+N m_{1}, \\
\frac{\mathrm{d} x_{2}}{\mathrm{~d} t}= & \frac{\sqrt{15}}{18} \tilde{\sigma}_{2} x_{1}+\sqrt{15}\left(\frac{1}{12} \tilde{\sigma}_{1}+\frac{1}{4} \tilde{\sigma}_{2}\right) x_{2}-\frac{\sqrt{15}}{60}\left(\tilde{\sigma}_{1}+\tilde{\sigma}_{2}\right) x_{3}+\frac{\sqrt{15}}{60}\left(\tilde{\sigma}_{1}+2 \tilde{\sigma}_{2}\right) x_{4}+N m_{2}, \\
\frac{\mathrm{d} x_{3}}{\mathrm{~d} t}= & -\frac{\sqrt{15}}{6}\left(\tilde{\sigma}_{1}+2 \tilde{\sigma}_{2}\right) x_{1}-\frac{\sqrt{15}}{6} \tilde{\sigma}_{2} x_{2}-\frac{\sqrt{15}}{12}\left(\tilde{\sigma}_{1}+2 \tilde{\sigma}_{2}\right) x_{3}+\left(1-\frac{\sqrt{15}}{12} \tilde{\sigma}_{2}\right) x_{4}+N m_{3}, \\
\frac{\mathrm{d} x_{4}}{\mathrm{~d} t}= & -\sqrt{15}\left(\frac{1}{6} \tilde{\sigma}_{1}+\frac{4}{9} \tilde{\sigma}_{2}\right) x_{1}-\sqrt{15}\left(\frac{2}{3} \tilde{\sigma}_{1}+\frac{3}{2} \tilde{\sigma}_{2}\right) x_{2}+\left(-1+\frac{\sqrt{15}}{36} \tilde{\sigma}_{2}\right) x_{3} \\
& -\frac{\sqrt{15}}{12}\left(\tilde{\sigma}_{1}+2 \tilde{\sigma}_{2}\right) x_{4}+N m_{4},
\end{aligned}
$$

where the third order nonlinear terms $\mathrm{Nm}_{i}(i=1,2,3,4)$ are omitted here.

Using an intrinsic method of harmonic analysis [23], we obtain the normal form of Eq.(35) as follows

$$
\begin{aligned}
& \frac{\mathrm{d} y_{1}}{\mathrm{~d} t}=y_{2}, \\
& \frac{\mathrm{d} y_{2}}{\mathrm{~d} t}=\frac{\sqrt{15}}{18} \tilde{\sigma}_{2} y_{1}+\frac{\sqrt{15}}{6}\left(\tilde{\sigma}_{1}+2 \tilde{\sigma}_{2}\right) y_{2}+\left(\frac{2 \sqrt{15}}{27}-\frac{10}{27}\right) y_{1}^{3}-\left(\frac{6}{5}+\frac{\sqrt{15}}{15}\right) y_{1} \rho^{2}, \\
& \frac{\mathrm{d} \rho}{\mathrm{d} t}=-\frac{\sqrt{15}}{12}\left(\tilde{\sigma}_{1}+2 \tilde{\sigma}_{2}\right) \rho-\left(\frac{1}{12}+\frac{\sqrt{15}}{5}\right) \rho^{3}-(64+40 \sqrt{15}) y_{1}^{2} \rho, \\
& \frac{\mathrm{d} \theta}{\mathrm{d} t}=1-\frac{\sqrt{15}}{18} \tilde{\sigma}_{2}-\left(\frac{7}{60}+\frac{\sqrt{15}}{10}\right) \rho^{2}+\left(\frac{8 \sqrt{15}}{9}-\frac{14}{3}\right) y_{1}^{2},
\end{aligned}
$$

where $y_{1}, y_{2}, \rho, \theta$ are the variables that are transformational systems which are topology equivalent to the original systems. The transformational systems can display the dynamical behaviors of the original ones. System (36) has the following equilibrium solutions.

(i) The initial equilibrium solution (E.S.) $y_{1}=y_{2}=\rho=0$.

Evaluating the Jacobian at the initial equilibrium solution, we obtain the stability conditions as follows 


$$
\tilde{\sigma}_{1}+2 \tilde{\sigma}_{2}<0, \quad \tilde{\sigma}_{2}>0,\left(15 \tilde{\sigma}_{1}^{2}+60 \tilde{\sigma}_{1} \tilde{\sigma}_{2}+8 \sqrt{15} \tilde{\sigma}_{2}+60 \tilde{\sigma}_{2}^{2}\right)<0,
$$

So the initial equilibrium solution is unstable.

(ii) The static bifurcation solution (S.B.)

$$
\begin{aligned}
& y_{1}^{2}=(\sqrt{15}+3) \tilde{\sigma}_{2} / 4, \\
& y_{2}=\rho=0,
\end{aligned}
$$

The stability conditions for this solution are as follows

$$
\tilde{\sigma}_{1}+2 \tilde{\sigma}_{2}<0, \quad \tilde{\sigma}_{2}>0,\left(15 \tilde{\sigma}_{1}^{2}+60 \tilde{\sigma}_{1} \tilde{\sigma}_{2}+32 \sqrt{15} \tilde{\sigma}_{2}+60 \tilde{\sigma}_{2}^{2}\right)>0,
$$

(iii) Hopf bifurcation solution (H.B.)

$$
\begin{aligned}
& y_{1}=y_{2}=0, \\
& \rho^{2}=-5 \sqrt{15}\left(\tilde{\sigma}_{1}+2 \tilde{\sigma}_{2}\right) /(5+12 \sqrt{15}) \approx-0.38\left(\tilde{\sigma}_{1}+2 \tilde{\sigma}_{2}\right),
\end{aligned}
$$

The stability conditions for this solution are as follows

$$
\begin{aligned}
& \tilde{\sigma}_{1}+2 \tilde{\sigma}_{2}<0,-16804.8 \tilde{\sigma}_{1}-40201 \tilde{\sigma}_{2}>0, \\
& 25620 \tilde{\sigma}_{2}^{2}-10086.33 \tilde{\sigma}_{2}+25620 \tilde{\sigma}_{1} \tilde{\sigma}_{2}-4216.28 \tilde{\sigma}_{1}+6405 \tilde{\sigma}_{1}^{2}>0,
\end{aligned}
$$

(iv) Hopf bifurcation solution 2 (H.B.2)

$$
\begin{aligned}
& y_{1}^{2}=-\left(0.0015 \tilde{\sigma}_{1}+0.0035 \tilde{\sigma}_{2}\right), \\
& y_{2}=0, \\
& \rho^{2}=0.000084 \tilde{\sigma}_{1}+0.153836 \tilde{\sigma}_{2},
\end{aligned}
$$

Obviously, when

$$
0.0015 \tilde{\sigma}_{1}+0.0035 \tilde{\sigma}_{2}<0 \text { and } 0.000084 \tilde{\sigma}_{1}+0.153836 \tilde{\sigma}_{2}>0
$$

there exists H.B.2 solution.

The stability conditions for this solution are

$$
\begin{aligned}
& -0.65 \tilde{\sigma}_{1}-1.04 \tilde{\sigma}_{2}>0, \\
& 0.34 \tilde{\sigma}_{2}^{3}+0.38 \tilde{\sigma}_{1} \tilde{\sigma}_{2}^{2}+0.11 \tilde{\sigma}_{1}^{2} \tilde{\sigma}_{2}-0.66 \tilde{\sigma}_{2}^{2}-0.28 \tilde{\sigma}_{1} \tilde{\sigma}_{2}>0, \\
& \left(0.0015 \tilde{\sigma}_{1}+0.0035 \tilde{\sigma}_{2}\right)\left(0.000084 \tilde{\sigma}_{1}+0.153836 \tilde{\sigma}_{2}\right)>0,
\end{aligned}
$$

so this bifurcation solution is unstable.

The bifurcation diagram is shown as in Fig 3. Here the dashed lines just mark the regions of different bifurcation solutions. From Fig 3 we can see that there exist stale non-trivial equilibrium solution and periodic motion under certain conditions. 


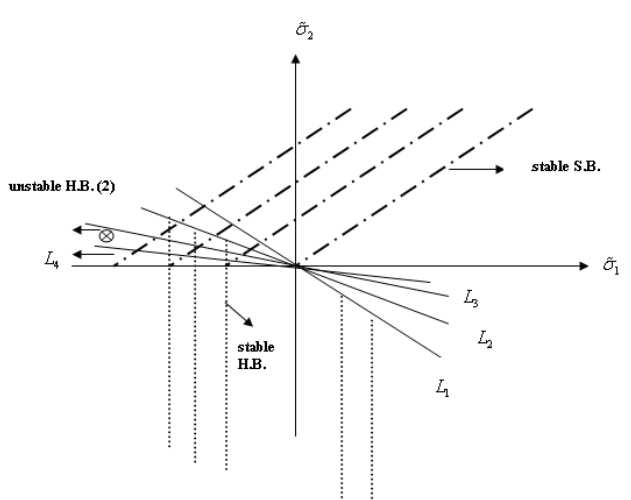

Figure 3. The bifurcation diagram in the case of double zero and a pair of purely imaginary eigenvalues.

\subsection{Case 3: two pairs of purely imaginary eigenvalues}

If the values of parameters are chosen as

$$
\begin{aligned}
& \mu=\left(\frac{1}{2}\right)^{1 / 4}, \quad C_{1 R}=1, \quad C_{2 R}=-1, \quad C_{1 I}=1, \quad C_{2 I}=1, H_{4 I}=1, H_{5 I}=-3, \\
& \alpha=0, \quad \sigma_{1}=\sigma_{2}=0, \quad H_{4 R}=H_{3 R}=H_{6 R}=H_{6 I}=0,
\end{aligned}
$$

then we have $R_{1}=R_{3}=0$ in Eq.(20) and the eigenvalues are

$$
\lambda_{1,2}= \pm \mathrm{i}, \quad \lambda_{3,4}= \pm \sqrt{2} \mathrm{i}
$$

Considering $\sigma_{1}, \sigma_{2}$ as parameters, using the parameter transformation $\sigma_{1}=\tilde{\sigma}_{1}$, $\sigma_{2}=\tilde{\sigma}_{2}$ and the state variable transformation

$$
\left[\begin{array}{l}
p_{1} \\
q_{1} \\
p_{2} \\
q_{2}
\end{array}\right]=\left[\begin{array}{cccc}
0 & \sqrt{2} & 0 & \frac{\sqrt{2}}{2} \\
0 & -1 & 0 & -1 \\
1 & -2^{-1 / 4}-2^{1 / 4} & \sqrt{2} & -2^{-1 / 4}-2^{-3 / 4} \\
-\sqrt{2} & 2^{1 / 4}-2^{-1 / 4} & -1 & 2^{-3 / 4}-2^{-1 / 4}
\end{array}\right]\left[\begin{array}{l}
x_{1} \\
x_{2} \\
x_{3} \\
x_{4}
\end{array}\right]
$$

Eq.(18) becomes

$$
\begin{aligned}
\frac{\mathrm{d} x_{1}}{\mathrm{~d} t}= & \left(\frac{\sqrt{2}}{2} \tilde{\sigma}_{1}-\frac{3 \sqrt{2}}{2} \tilde{\sigma}_{2}\right) x_{1}+\left(1+\left(2^{-1 / 4}+2^{5 / 4}\right) \tilde{\sigma}_{2}\right) x_{2}+\left(\frac{3}{4} \tilde{\sigma}_{1}-\frac{9}{4} \tilde{\sigma}_{2}\right) x_{3} \\
& +3 \cdot 2^{-3 / 4} \tilde{\sigma}_{2} x_{4}+N h_{1}, \\
\frac{\mathrm{d} x_{2}}{\mathrm{~d} t}= & -x_{1}+\frac{\sqrt{2}}{2}\left(\tilde{\sigma}_{1}+\tilde{\sigma}_{2}\right) x_{2}+\frac{3 \sqrt{2}}{8}\left(\tilde{\sigma}_{1}+\tilde{\sigma}_{2}\right) x_{4}+N h_{2}, \\
\frac{\mathrm{d} x_{3}}{\mathrm{~d} t}= & \left(\frac{9}{4} \tilde{\sigma}_{2}-\frac{3}{4} \tilde{\sigma}_{1}\right) x_{1}-3 \cdot 2^{-1 / 4} \tilde{\sigma}_{2} x_{2}+\left(\frac{3 \sqrt{2}}{2} \tilde{\sigma}_{2}-\frac{\sqrt{2}}{2} \tilde{\sigma}_{1}\right) x_{3} \\
& +\left(\sqrt{2}+\left(2^{-3 / 4}-2^{3 / 4}\right) \tilde{\sigma}_{2} x_{4}+N h_{3},\right. \\
\frac{\mathrm{d} x_{4}}{\mathrm{~d} t}= & -\frac{3 \sqrt{2}}{4}\left(\tilde{\sigma}_{1}+\tilde{\sigma}_{2}\right) x_{2}-\sqrt{2} x_{3}-\frac{\sqrt{2}}{2}\left(\tilde{\sigma}_{1}+\tilde{\sigma}_{2}\right) x_{4}+N h_{4},
\end{aligned}
$$

The third order nonlinear terms $N h_{i}(i=1,2,3,4)$ are also omitted here. 
With the method of multiple scales and computer algebra [24], we get the normal form of Eq.(47) in polar coordinates as follows:

$$
\begin{aligned}
& \dot{r}_{1}=r_{1}\left(\alpha_{11} \tilde{\sigma}_{1}+\alpha_{12} \tilde{\sigma}_{2}+a_{20} r_{1}^{2}+a_{02} r_{2}^{2}\right), \\
& \dot{r}_{2}=r_{2}\left(\alpha_{21} \tilde{\sigma}_{1}+\alpha_{22} \tilde{\sigma}_{2}+b_{20} r_{1}^{2}+b_{02} r_{2}^{2}\right), \\
& \dot{\theta}_{1}=\omega_{1 c}+\beta_{11} \tilde{\sigma}_{1}+\beta_{12} \tilde{\sigma}_{2}+c_{20} r_{1}^{2}+c_{2} r_{2}^{2}, \\
& \dot{\theta}_{2}=\omega_{2 c}+\beta_{21} \tilde{\sigma}_{1}+\beta_{22} \tilde{\sigma}_{2}+d_{20} r_{1}^{2}+d_{02} r_{2}^{2},
\end{aligned}
$$

where $\alpha_{11}=\alpha_{22}=\frac{\sqrt{2}}{2}, \alpha_{12}=\alpha_{21}=-\frac{\sqrt{2}}{2}, \quad \beta_{11}=\beta_{21}=0, \quad \beta_{12}=2^{-5 / 4}+2^{1 / 4}$,

$\beta_{22}=2^{-7 / 4}-2^{-1 / 4}, \quad a_{20}=\frac{35}{8}-\frac{9}{8} \cdot 2^{3 / 4}+\frac{9}{2} \cdot 2^{1 / 4}-\frac{7 \sqrt{2}}{8}, a_{02}=\frac{9}{2} \cdot 2^{1 / 4}-\frac{3 \sqrt{2}}{4}-\frac{7}{8} \cdot 2^{3 / 4}+\frac{33}{8}$,

$b_{20}=-7-\frac{61}{8} \cdot 2^{3 / 4}-\frac{81}{16} \sqrt{2}+\frac{5}{8} \cdot 2^{3 / 4}, \quad b_{02}=-\frac{31}{16}+\frac{9}{32} \cdot 2^{3 / 4}-\frac{111 \sqrt{2}}{64}-\frac{71}{32} \cdot 2^{1 / 4}$,

$c_{20}=\frac{9}{2}+\frac{11}{2} \cdot 2^{1 / 4}-\frac{27 \sqrt{2}}{8}+\frac{115}{15} \cdot 2^{3 / 4}, \quad c_{02}=\frac{63}{8} \cdot 2^{1 / 4}+\frac{45}{8} \cdot 2^{3 / 4}-\frac{13 \sqrt{2}}{8}+\frac{13}{2}$,

$d_{20}=-\frac{13}{8}-\frac{7}{8} \cdot 2^{1 / 4}-\frac{55 \sqrt{2}}{16}-\frac{41}{16} \cdot 2^{3 / 4}, \quad d_{02}=-\frac{33}{64}-\frac{69}{64} \cdot 2^{3 / 4}-\frac{99 \sqrt{2}}{64}$,

and $r_{1}, r_{2}, \theta_{1}$ and $\theta_{2}$ are the variables that are transformational systems which are topology equivalent to the original systems.

We now discuss the main types of equilibrium states and their stability for system (48). These fall into four categories.

(i) Trivial state: The initial equilibrium solution (E.S.): $r_{1}=r_{2}=0$.

Evaluating the Jacobi matrix at the initial equilibrium solution results in the stability conditions for the E.S. as $\alpha_{11} \tilde{\sigma}_{1}+\alpha_{12} \tilde{\sigma}_{2}<0$ and $\alpha_{21} \tilde{\sigma}_{1}+\alpha_{22} \tilde{\sigma}_{2}<0$,i.e., $\tilde{\sigma}_{1}-\tilde{\sigma}_{2}<0$ and $\tilde{\sigma}_{2}-\tilde{\sigma}_{1}<0$, so it is unstable.

(ii) Pure mode 1: Hopf bifurcation solution (i.e., a self-sustained oscillation, H.B.(I) with frequency $\omega_{1}$ ):

$$
\begin{aligned}
& r_{1}^{2}=-\frac{1}{a_{20}}\left(\alpha_{11} \tilde{\sigma}_{1}+\alpha_{12} \tilde{\sigma}_{2}\right)=-0.11 \tilde{\sigma}_{1}+0.11 \tilde{\sigma}_{2}, \\
& r_{2}=0, \\
& \omega_{1}=\omega_{1 c}+\beta_{11} \tilde{\sigma}_{1}+\beta_{12} \tilde{\sigma}_{2}+c_{20} r_{1}^{2}=1-2.02 \tilde{\sigma}_{1}+3.63 \tilde{\sigma}_{2}
\end{aligned}
$$

Obviously, when $\tilde{\sigma}_{2}-\tilde{\sigma}_{1}>0$ there exists H.B.(I) solution.

Evaluating the Jacobi matrix at the H.B.(I) solution results in the following

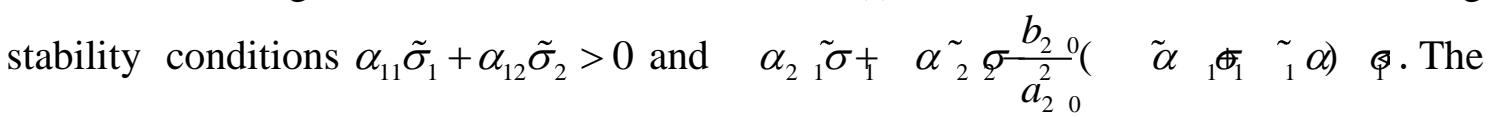
condition $\alpha_{11} \tilde{\sigma}_{1}+\alpha_{12} \tilde{\sigma}_{2}>0$ implies that $\tilde{\sigma}_{1}-\tilde{\sigma}_{2}>0$, So H.B.(I) solution is unstable.

(iii) Pure mode 2: Hopf bifurcation solution (H.B.(II) with frequency $\omega_{2}$ )

$$
r_{1}=0 \text {, }
$$




$$
\begin{aligned}
& r_{2}^{2}=-\frac{1}{b_{02}}\left(\alpha_{21} \tilde{\sigma}_{1}+\alpha_{22} \tilde{\sigma}_{2}\right)=-0.11 \tilde{\sigma}_{1}+0.11 \tilde{\sigma}_{2}, \\
& \omega_{2}=\omega_{2 c}+\beta_{21} \tilde{\sigma}_{1}+\beta_{22} \tilde{\sigma}_{2}+d_{02} r_{2}^{2}=\sqrt{2}+0.49 \tilde{\sigma}_{1}-1.03 \tilde{\sigma}_{2},
\end{aligned}
$$

Obviously, when $\tilde{\sigma}_{2}-\tilde{\sigma}_{1}>0$ there exists H.B.(II) solution.

Evaluating the Jacobi matrix at the H.B.(II) solution results the following stability conditions $\alpha_{21} \tilde{\sigma}_{1}+\alpha_{22} \tilde{\sigma}_{2}>0$ and $\alpha_{11} \tilde{\sigma}_{1}+\alpha_{12} \tilde{\sigma}_{2}-\frac{a_{02}}{b_{02}}\left(\alpha_{21} \tilde{\sigma}_{1}+\alpha_{22} \tilde{\sigma}_{2}\right)<0$,i.e., $\tilde{\sigma}_{2}-\tilde{\sigma}_{1}>0$ and $\tilde{\sigma}_{2}-\tilde{\sigma}_{1}<0$, so H.B.(II) solution is also unstable.

(iv) Mixed modes: quasi-periodic solution (2D tori with frequency $\omega_{1}, \omega_{2}$ ):

$$
\begin{aligned}
& r_{1}^{2}=\frac{a_{02}\left(\alpha_{21} \tilde{\sigma}_{1}+\alpha_{22} \tilde{\sigma}_{2}\right)-b_{02}\left(\alpha_{11} \tilde{\sigma}_{1}+\alpha_{12} \tilde{\sigma}_{2}\right)}{a_{20} b_{02}-a_{02} b_{20}}=-0.0025 \tilde{\sigma}_{1}+0.0025 \tilde{\sigma}_{2}, \\
& r_{2}^{2}=\frac{b_{20}\left(\alpha_{21} \tilde{\sigma}_{1}+\alpha_{12} \tilde{\sigma}_{2}\right)-a_{20}\left(\alpha_{21} \tilde{\sigma}_{1}+\alpha_{22} \tilde{\sigma}_{2}\right)}{a_{20} b_{02}-a_{02} b_{20}}=0.18 \tilde{\sigma}_{1}+0.1 \tilde{\sigma}_{2}, \\
& \omega_{1}=\omega_{1 c}+\beta_{11} \tilde{\sigma}_{1}+\beta_{12} \tilde{\sigma}_{2}+c_{20} r_{1}^{2}+c_{02} r_{2}^{2}=1+4.18 \tilde{\sigma}_{1}+3.95 \tilde{\sigma}_{2}, \\
& \omega_{2}=\omega_{2 c}+\beta_{21} \tilde{\sigma}_{1}+\beta_{22} \tilde{\sigma}_{2}+d_{20} r_{1}^{2}+d_{02} r_{2}^{2}=\sqrt{2}+4.25 \tilde{\sigma}_{1}+1.73 \tilde{\sigma}_{2}
\end{aligned}
$$

Obviously, when $\tilde{\sigma}_{2}-\tilde{\sigma}_{1}>0$ and $0.18 \tilde{\sigma}_{1}+0.1 \tilde{\sigma}_{2}>0$, there exists $2 \mathrm{D}$ tori.

The stability conditions for the quasi-periodic solutions are obtained from the trace and determinant of the Jacobian, given by

$$
\begin{aligned}
\operatorname{Tr} & =2\left(a_{20} r_{1}^{2}+b_{02} r_{2}^{2}\right) \\
& =\frac{2\left[a_{20}\left(a_{02}-b_{02}\right)\left(\alpha_{21} \delta_{1}+\alpha_{22} \delta_{2}\right)-b_{02}\left(a_{20}-b_{20}\right)\left(\alpha_{11} \delta_{1}+\alpha_{12} \delta_{2}\right)\right]}{a_{20} b_{02}-a_{02} b_{20}}<0, \\
\text { Det } & =4\left(a_{20} b_{02}-a_{02} b_{20}\right) r_{1}^{2} r_{2}^{2}>0,
\end{aligned}
$$

i.e., $-1.21 \tilde{\sigma}_{1}-0.64 \tilde{\sigma}_{2}<0$. The critical lines are illustrated in Fig 4. Here the dashed lines also just mark the regions of different bifurcation solutions. From Fig 4 one can see that there exist stable mixed modes quasi-periodic motions under certain conditions in this case.

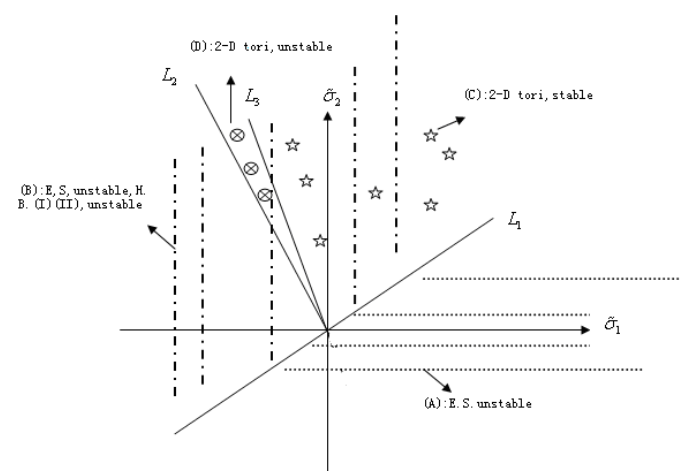

Figure 4 . The bifurcation diagram in the case of two pairs of purely imaginary eigenvalues. 


\section{NUMERICAL SIMULATIONS}

In this section, with the fourth-order Runge-Kutta method, the phase portraits of system (14) are obtained for different values $\tilde{\sigma}_{1}$ and $\tilde{\sigma}_{2}$.

For case 1 , choosing $\left(\tilde{\sigma}_{1}, \tilde{\sigma}_{2}\right)=(0.05,0.15), \quad\left(\tilde{\sigma}_{1}, \tilde{\sigma}_{2}\right)=(-0.05,0.1)$, $\left(\tilde{\sigma}_{1}, \tilde{\sigma}_{2}\right)=(0.05,0.01)$, respectively, we obtain the phase portraits of system (2.14) as in Fig 5-Fig 7.

Notice that these parameters of $\left(\tilde{\sigma}_{1}, \tilde{\sigma}_{2}\right)$ are in the stable regions of the initial equilibrium solution, static bifurcation solution and limit cycle, respectively, numerical results agree with the analytical ones.

For case 2 , choosing $\left(\tilde{\sigma}_{1}, \tilde{\sigma}_{2}\right)=(0.05,0.05)$ (in the stable region of the static bifurcation solution), $\left(\tilde{\sigma}_{1}, \tilde{\sigma}_{2}\right)=(-0.05,-0.1)$ (in the stable region of the Hopf bifurcation solution), respectively, we obtain the phase portraits of system (2.14) as in Fig 8-Fig 9.

For case 3 , choosing $\left(\tilde{\sigma}_{1}, \tilde{\sigma}_{2}\right)=(0.05,0.05)$ (in the stable region of 2-D tori), the phase portraits are shown as in Fig 10.

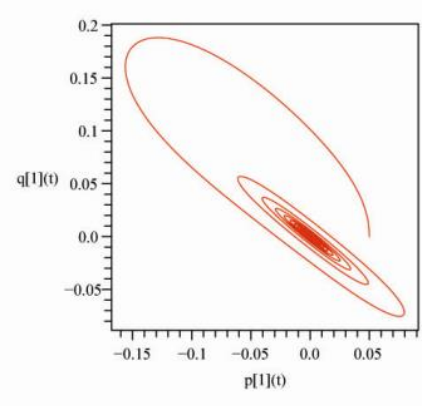

(a)

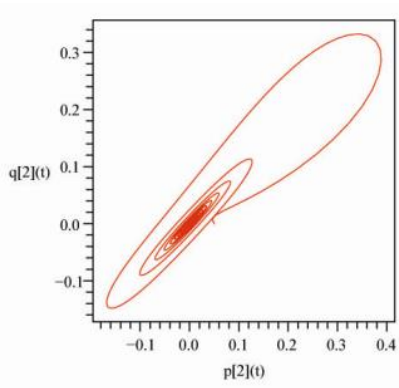

(b)

Figure 5. Trajectory starting from $\left(p_{1}, q_{1}, q_{1}, q_{2}\right)=(0.05,0,0.05,0)$ converges to the E.S. for case 1

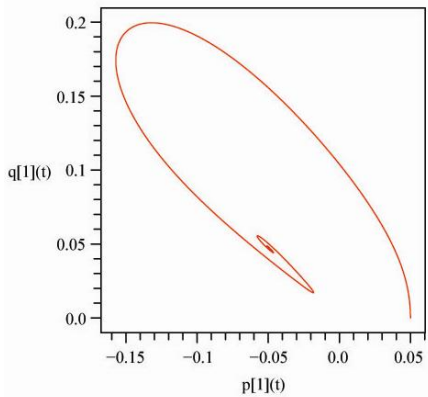

(a)

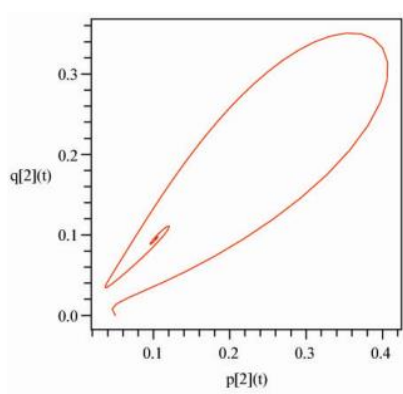

(b)

Figure 6. Trajectory starting from $\left(p_{1}, q_{1}, q_{1}, q_{2}\right)=(0.05,0,0.05,0)$ converges to the S.B. for case 1 


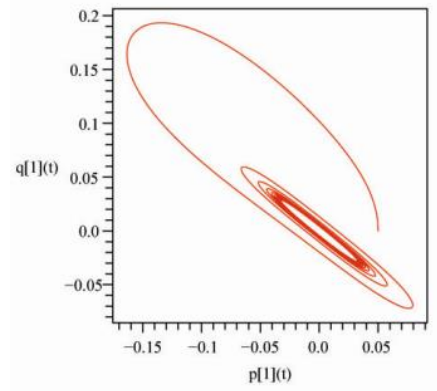

(a)

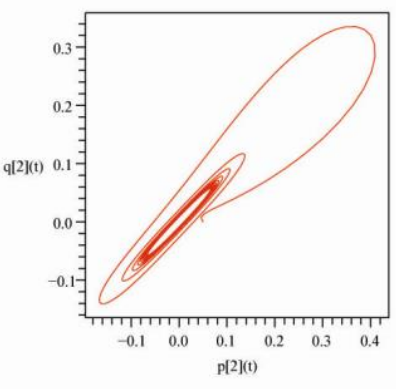

(b)

Figure 7. Trajectory starting from $\left(p_{1}, q_{1}, q_{1}, q_{2}\right)=(0.05,0,0.05,0)$ converges to the L.C. for case 1

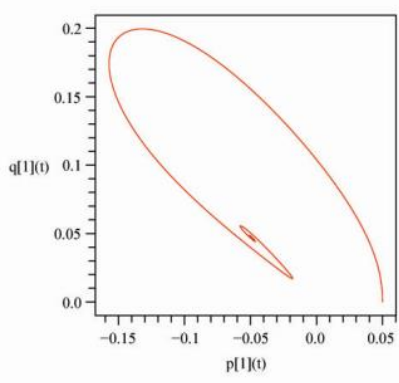

(a)

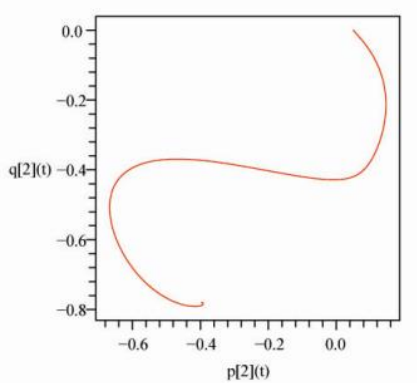

(b)

Figure 8 . Trajectory starting from $\left(p_{1}, q_{1}, q_{1}, q_{2}\right)=(0.05,0,0.05,0)$ converges to the S.B. for case 2

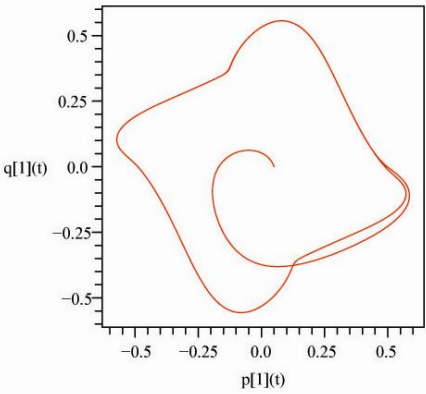

(a)

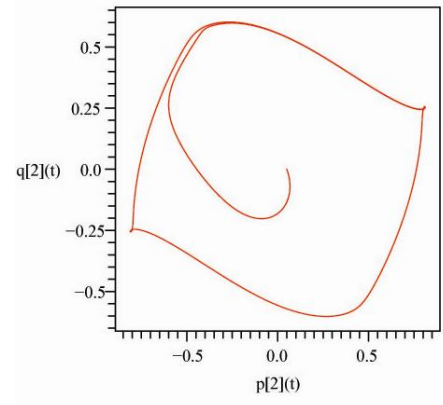

(b)

Figure 9. Trajectory starting from $\left(p_{1}, q_{1}, q_{1}, q_{2}\right)=(0.05,0,0.05,0)$ converges to the H.B. for case 2

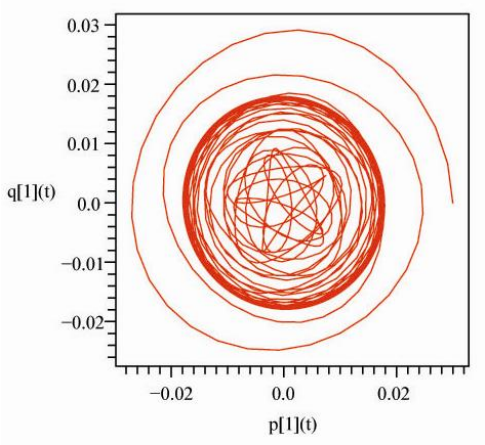

(a)

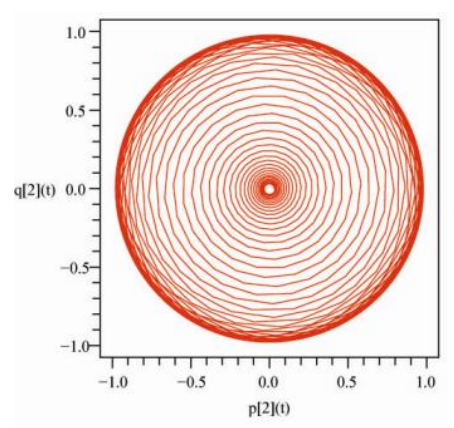

(b)

Figure 10. Trajectory starting from $\left(p_{1}, q_{1}, q_{1}, q_{2}\right)=(0.05,0,0.05,0)$ converges to the 2 -D tori for case 3 


\section{CONCLUSIONS}

With the method of normal forms, the bifurcation solutions and their stability of a hinged-hinged pipe conveying pulsating fluid with combination parametric and internal resonances are studied in detail. When the stability conditions for the initial equilibrium solutions are not satisfied, bifurcations including pitchfork bifurcation, Hopf bifurcation, 2-D tori may occur. Complicated dynamical phenomena of this model are presented here. Numerical simulations agree with the analytical results.

\section{ACKNOWLEDGMENTS}

This research was supported by the National Natural Science Foundation of China (Nos.11202095, 11172125), China Postdoctoral Science Foundation (No. 2013T60531), and National Research Foundation for the Doctoral Program of Higher Education of China (20133218110025).

\section{APPENDIX}

$$
\begin{aligned}
& J_{11}=J_{12}=J_{14}=J_{23}=1, \quad J_{13}=\frac{\sqrt{6 \sqrt{13}+6}}{12}(\sqrt{13}-7), \quad J_{21}=J_{22}=-\frac{\sqrt{6 \sqrt{13}+6}}{6} \text {, } \\
& J_{24}=J_{31}=-\frac{\sqrt{6 \sqrt{13}+6}}{12}(\sqrt{13}+1), \quad J_{32}=-\frac{\sqrt{6 \sqrt{13}+6}}{6}-\frac{\sqrt{6 \sqrt{13}+6}}{12}(\sqrt{13}+1) \text {, } \\
& J_{33}=2, \quad J_{34}=\frac{\sqrt{6 \sqrt{13}+6}}{6}(\sqrt{13}+1), \quad J_{41}=-\frac{7}{6}-\frac{\sqrt{13}}{6}, \quad J_{43}=\frac{2}{3} \sqrt{6 \sqrt{13}+6}, \\
& J_{42}=\frac{5-\sqrt{13}}{12}+\frac{(\sqrt{13}-5)(4+\sqrt{13})}{6}, \quad J_{44}=-\frac{2}{3} \sqrt{13}-\frac{14}{3}, \\
& a_{11}=\frac{(5 \sqrt{13}+113) \tilde{\sigma}_{1}+(25 \sqrt{13}-107) \tilde{\sigma}_{2}}{4 \sqrt{6 \sqrt{13}+6}(2 \sqrt{13}-5)}, a_{12}=1+\frac{(63 \sqrt{13}+255) \tilde{\sigma}_{1}-(21 \sqrt{13}+117) \tilde{\sigma}_{2}}{4 \sqrt{6 \sqrt{13}+6}(2 \sqrt{13}-5)}, \\
& a_{13}=\frac{(7 \sqrt{13}-32) \tilde{\sigma}_{1}+(16-9 \sqrt{13}) \tilde{\sigma}_{2}}{2(2 \sqrt{13}-5)}, \quad a_{14}=-\frac{(17 \sqrt{13}+35) \tilde{\sigma}_{1}-(95 \sqrt{13}+461) \tilde{\sigma}_{2}}{2 \sqrt{6 \sqrt{13}+6}(2 \sqrt{13}-5)}, \\
& a_{21}=-\frac{3\left[(\sqrt{13}+7) \tilde{\sigma}_{1}+(5 \sqrt{13}-13) \tilde{\sigma}_{2}\right]}{2 \sqrt{6 \sqrt{13}+6}(2 \sqrt{13}-5)}, \quad a_{22}=-\frac{3\left[(7 \sqrt{13}+11) \tilde{\sigma}_{1}+(3 \sqrt{13}-9) \tilde{\sigma}_{2}\right]}{4 \sqrt{6 \sqrt{13}+6}(2 \sqrt{13}-5)}, \\
& a_{23}=-\frac{3\left[(\sqrt{13}-5) \tilde{\sigma}_{1}+(7-3 \sqrt{13}) \tilde{\sigma}_{2}\right]}{2(2 \sqrt{13}-5)}, \quad a_{24}=\frac{3\left[(\sqrt{13}+1) \tilde{\sigma}_{1}-(7 \sqrt{13}+31) \tilde{\sigma}_{2}\right]}{\sqrt{6 \sqrt{13}+6}(2 \sqrt{13}-5)}, \\
& a_{31}=\frac{3\left[(\sqrt{13}+1) \tilde{\sigma}_{1}-(\sqrt{13}+1) \tilde{\sigma}_{2}\right]}{8(2 \sqrt{13}-5)}, a_{32}=\frac{9\left[(\sqrt{13}+3) \tilde{\sigma}_{1}-(\sqrt{13}+3) \tilde{\sigma}_{2}\right]}{16(2 \sqrt{13}-5)}, \\
& a_{33}=-1+\frac{(3 \sqrt{13}-12) \tilde{\sigma}_{1}-\tilde{\sigma}_{2}}{\sqrt{6 \sqrt{13}++6}(2 \sqrt{13}-5)}, \quad a_{34}=\frac{-3\left[(\sqrt{13}+4) \tilde{\sigma}_{1}-(7 \sqrt{13}+16) \tilde{\sigma}_{2}\right]}{4(2 \sqrt{13}-5)},
\end{aligned}
$$




$$
\begin{aligned}
& a_{41}=-\frac{(\sqrt{13}-2) \tilde{\sigma}_{1}+(2-\sqrt{13}) \tilde{\sigma}_{2}}{6 \sqrt{6 \sqrt{13}+6}(2 \sqrt{13}-5)}, \quad a_{42}=\frac{-3\left[(\sqrt{13}+1) \tilde{\sigma}_{1}+(3-\sqrt{13}) \tilde{\sigma}_{2}\right]}{8 \sqrt{6 \sqrt{13}+6}(2 \sqrt{13}-5)}, \\
& a_{43}=\frac{(\sqrt{13}-2) \tilde{\sigma}_{1}+(\sqrt{13}-6) \tilde{\sigma}_{2}}{4(2 \sqrt{13}-5)}, \quad a_{44}=-3+\frac{(\sqrt{13}-8) \tilde{\sigma}_{1}+(29-4 \sqrt{13}) \tilde{\sigma}_{2}}{\sqrt{6 \sqrt{13}+6}(2 \sqrt{13}-5)},
\end{aligned}
$$

\section{REFERENCES}

1.M. P. Paidoussis, Flow-induced instabilities of cylindrical structures, Applied Mechanics Re-view 40, 163-175, 1987.

2. M. P. Paidoussis and D. X. Li, Pipes conveying uid: a model dynamical problem, Journal of Fluids and Structures 7, 137-204, 1993.

3. M. P. Paidoussis, Fluid-Structure Interactions Slender Structure and Axial Flow, Academic Press, London, 1998.

4.S. S. Chen, Dynamic stability of a tube conveying uid, ASCE Journal of Engineering Mechanics 97, 1469-1485, 1971.

5. M. P. Paidoussis and N. T. Issid, "Dynamic stability of pipes conveying fluid," Journal of Sound and Vibration 33, 267-294, 1974.

6. M. P. Paidoussis and C. Sundararajan, Parametric and combination resonances of a pipe conveying pulsating fluid, ASME Journal of Applied Mechanics 42, 780-784,1974.

7. J. Ginsberg, The dynamic stability of a pipe conveying a pulsatile flow, International Journal of Engineering Science 11, 1013-1024, 1973.

8. S. T. Ariartnam and N.S. Namachchivaya, Dynamic stability of pipes conveying pulsating fluid, Journal of Sound and Vibration 107, 215-230, 1986.

9. K. Jayaraman and W. M. Tien, Chaotic oscillators in pipes conveying pulsating fluid, Nonlinear Dynamics 10, 333-357, 1996.

10. L. N. Panda and R. C. Kar, Nonlinear dynamics of a pipe conveying pulsating fluid with combination principle parametric and internal resonances, Journal of Sound and Vibration, 309, 375-406, 2008.

11. L. N. Panda and R. C. Kar, Nonlinear dynamics of a pipe conveying pulsating fluid with parametric and internal resonances, Nonlinear Dynamics 49, 9-30, 2007.

12. L. Wang, A further study on the non-linear dynamics of simply supported pipes conveying pulsating fluid, International Journal of Non-Linear Mechanics 44, 115-121, 2009.

13. J. D. Jin and Z.Y. Song, Parametric resonances of supported pipes conveying pulsating fluid, Journal of Fluids and Structures 20, 763-783, 2005.

14. Y. Modarres-Sadeghi and M.P.Paldoussis, Nonlinear dynamics of extensible fluidconveying pipes, supported at both ends, Journal of Fluids and Structures 25, 535-543, 2009.

15. L. Wang and Q. Ni, A note on the stability and chaotic motions of a restrained pipe conveying fluid, Journal of Sound and Vibration 296, 1079-1083, 2006.

16. U. Lee, and J. Park, Spectral element modeling and analysis of a pipeline conveying internal unsteady fluid, Journal of Fluids and Structures 22, 273-292, 2006.

17. R.J. McDonald and N. Sri Namachchivaya, Pipes conveying pulsating fluid near a 
0: 1 resonance: Global bifurcations, Journal of Fluids and Structures 21, 665-687, 2005.

18. J.D. Jin and G.S. Zou, Bifurcations and chaotic motions in the autonomous system of a restrained pipe conveying fluid, Journal of Sound and Vibration 260, 783-805, 2003.

19. S.I. Lee and J. Chung, New nonlinear modeling for vibration analysis of a straight pipe conveying fluid, Journal of Sound and Vibration 54, 313-325, 2002.

20. B.G.Sinir, Bifurcation and chaos of slightly curved pipes, Mathematical and Computational Applications 15, 490-502, 2010.

21. P.Yu and Q. S. Bi, Analysis of non-linear dynamics and bifurcations of a double pendulum, Journal of Sound and Vibration 217, 691-736, 1998.

22. P. Yu and K. Huseyin, Static and dynamic bifurcations associated with a doublezero eigenvalue, Dynamics and Stability of Systems 1, 1-42, 1986.

23. P. Yu and R. Huseyin, Bifurcations associated with a double zero and a pair of pure imaginary eigenvalues, SIAM Journal on Applied Mathematics 48, 229-261, 1988.

24. P. Yu, Analysis on double Hopf bifurcation using computer algebra with the aid of multiple scales, Nonlinear Dynamics 27, 19-53, 2002. 